\title{
Clusters AgeS Experiment. New variable stars in the globular cluster M 22
}

\author{
J. Kaluzny ${ }^{1}$ and I. B. Thompson ${ }^{2}$ \\ 1 Copernicus Astronomical Center, Bartycka 18, 00-716 Warsaw, Poland \\ 2 Carnegie Observatories, 813 Santa Barbara St., Pasadena, CA 91101-1292, USA \\ e-mail: ian@ociw.edu
}

Received 6 February 2001 / Accepted 23 April 2001

\begin{abstract}
We report the identification of 36 new variable stars located in the central field of the globular cluster M 22. The sample includes 10 SX Phe stars, 7 RR Lyr stars and 17 candidate eclipsing binaries. A low-amplitude variable was detected among the extended horizontal branch stars.
\end{abstract}

Key words. globular clusters: individual: M 22 - stars: variables: RRlyr; general - blue stragglers; binaries: eclipsing

\section{Introduction}

The Cluster AgeS Experiment (CASE) is a long term project aimed at the determination of ages and distances of globular clusters using observations of detached eclipsing binary stars (Paczyński 1997; Thompson et al. 2001). Several southern clusters are being surveyed for eclipsing binaries with the 1-m Swope telescope while follow-up observations of selected variables are made with the $2.5-\mathrm{m}$ du Pont telescope. During an observing run in June 1999 on the du Pont telescope we monitored the central part of the globular cluster M 22 for 4.7 hours. In this contribution we report on the properties of several newly discovered short period variables.

M 22 (NGC $6656 ; l=9.9 \mathrm{deg}, b=-7.6 \mathrm{deg}$ ) is a bright and rich globular cluster projected against the outer parts of the galactic bulge. Although M 22 ranks 4th among all globulars in heliocentric distance, studies of this cluster have been hampered by the large density of field stars and patchy reddening. The mean value of the reddening is estimated to be $E(B-V)=0.38$ (Richter et al. 1999). Sawyer Hogg's catalogue and its recent update (Clement 1997) list 35 variable stars as possible members of the cluster, all either RR Lyr stars or red long-period variables. Eight additional variables were identified in the cluster halo by Kravtsov et al. (1994). These authors list equatorial coordinates of the 43 variables thought to be cluster members.

Send offprint requests to: J. Kaluzny, e-mail: jka@camk.edu.pl

\section{Observations and data reduction}

The data were obtained with the $2 \mathrm{~K} \times 2 \mathrm{~K}$ pixel TEK\#5 CCD camera on the 2.5-m du Pont telescope at Las Campanas Observatory on the night of June 9/10, 1999. The field of view was $8.9 \times 8.9 \operatorname{arcmin}^{2}$ with a scale of 0.26 arcsec pixel $^{-1}$. An area centered on the cluster center was imaged through $B$ and $V$ filters for a period of 4.7 hours. A total of 76 images were taken through the $V$ filter with exposure times ranging from $30 \mathrm{~s}$ to $60 \mathrm{~s}$, while exposure times for the 33 images in the $B$-band ranged from $60 \mathrm{~s}$ to $80 \mathrm{~s}$. The $F W H M$ measured for individual frames was between 0.91 and 1.43 arcsec with a median value of 1.31 arcsec for the $V$ filter. The sky was clear during the observations.

The preliminary processing of the CCD frames was done with the IRAF-CCDPROC package ${ }^{1}$. We used the ISIS-2.1 image subtraction package (Alard \& Lupton 1998; Alard 2000) to detect the variable stars and to extract their light curves. We followed the prescription given in the ISIS.V2 manual to obtain differential light curves expressed in ADU units. The template image for the $V$ filter was constructed from the 7 frames of the best image quality, while the $B$-band template was constructed from 14 frames. Magnitude zero points for the ISIS differential light curves were measured from the template images using the DAOPHOT/ALLSTAR software package (Stetson 1987). Transformation from the instrumental system to the standard $B V$ system was accomplished by

\footnotetext{
${ }^{1}$ IRAF is distributed by the National Optical Astronomy Observatories, which are operated by the Association of Universities for Research in Astronomy, Inc., under cooperative agreement with the NSF.
} 
Table 1. J2000.0 equatorial coordinates of new variables.

\begin{tabular}{llll|llll}
\hline Name & Ra[h:m:s] & Dec $\left[^{\circ}::^{\prime \prime}\right]$ & Type & Name & Ra[h:m:s] & Dec[$\left[:^{\prime}:^{\prime \prime}\right]$ & Type \\
\hline CASE M22_01 & $18: 36: 43.34$ & $-23: 56: 24.9$ & Ecl/EW & CASE M22_28 & $18: 36: 22.04$ & $-23: 52: 05.9$ & SX \\
CASE M22_02 & $18: 36: 41.82$ & $-23: 56: 20.3$ & Ecl/EB & CASE M22_29 & $18: 36: 20.97$ & $-23: 55: 47.5$ & SX \\
CASE M22_03 & $18: 36: 41.27$ & $-23: 52: 19.2$ & Ecl/EW & CASE M22_33 & $18: 36: 16.86$ & $-23: 53: 54.5$ & Ecl/EW \\
CASE M22_04 & $18: 36: 39.38$ & $-23: 52: 25.1$ & SX & CASE M22_34 & $18: 36: 16.90$ & $-23: 55: 24.3$ & SX \\
CASE M22_05 & $18: 36: 39.09$ & $-23: 50: 27.3$ & SX & CASE M22_36 & $18: 36: 15.90$ & $-23: 56: 06.3$ & RR \\
CASE M22_07 & $18: 36: 36.85$ & $-23: 56: 59.9$ & Ecl/EW & CASE M22_37 & $18: 36: 13.19$ & $-23: 53: 46.3$ & RR \\
CASE M22_08 & $18: 36: 35.68$ & $-23: 55: 14.6$ & Ecl/EW & CASE M22_38 & $18: 36: 11.45$ & $-23: 56: 46.8$ & SX \\
CASE M22_10 & $18: 36: 32.30$ & $-23: 54: 27.2$ & SX & CASE M22_39 & $18: 36: 10.00$ & $-23: 51: 57.3$ & Ecl/EW \\
CASE M22_12 & $18: 36: 30.95$ & $-23: 53: 48.3$ & RR-field & CASE M22_40 & $18: 36: 08.14$ & $-23: 51: 48.5$ & Ecl/EW \\
CASE M22_13 & $18: 36: 30.86$ & $-23: 53: 45.6$ & Ecl/EW & CASE M22_41 & $18: 36: 07.10$ & $-23: 54: 12.6$ & Ecl/EW \\
CASE M22_14 & $18: 36: 30.67$ & $-23: 53: 53.1$ & RR & CASE M22_42 & $18: 36: 34.67$ & $-23: 52: 30.5$ & Puls? \\
CASE M22_15 & $18: 36: 30.13$ & $-23: 49: 58.7$ & Ecl/EW & CASE M22_43 & $18: 36: 24.27$ & $-23: 56: 18.9$ & Ecl/EW \\
CASE M22_16 & $18: 36: 30.37$ & $-23: 57: 12.3$ & RR & CASE M22_45 & $18: 36: 22.04$ & $-23: 54: 41.0$ & SX \\
CASE M22_18 & $18: 36: 29.04$ & $-23: 49: 58.8$ & Ecl/EA & CASE M22_46 & $18: 36: 21.78$ & $-23: 58: 24.3$ & Ecl/EB \\
CASE M22_20 & $18: 36: 26.09$ & $-23: 51: 26.4$ & Ecl/EW & CASE M22_48 & $18: 36: 16.55$ & $-23: 57: 35.7$ & Ecl? \\
CASE M22_23 & $18: 36: 23.84$ & $-23: 51: 15.8$ & Ecl/EW & CASE M22_51 & $18: 36: 35.08$ & $-23: 53: 03.6$ & sdB \\
CASE M22_26 & $18: 36: 23.17$ & $-23: 53: 22.7$ & RR & CASE M22_54 & $18: 36: 24.95$ & $-23: 50: 49.7$ & SX \\
CASE M22_27 & $18: 36: 22.54$ & $-23: 55: 12.4$ & SX & CASE M22_55 & $18: 36: 23.25$ & $-23: 53: 57.3$ & RR \\
\hline
\end{tabular}

using the following relations:

$$
\begin{aligned}
& V=v-0.018(B-V)-2.998 \\
& B-V=0.948(b-v)+0.311
\end{aligned}
$$

where $v$ and $b$ are instrumental magnitudes. The color terms are based on observations of several Landolt fields (Landolt 1992) obtained on nights other than when M 22 was monitored. The zero points of the transformation were derived using a set of 16 local standards with photographic photometry published by Alcaino \& Liller $(1983)^{2}$. The $(B-V)$ colors of these local standards range from 0.33 to 1.11 .

Due to this approximate calibration of our photometry, it is rather difficult to assess with confidence the accuracy of the zero points for $V$ and $B-V$. We note however, that the relative locations of the variables on the cluster color-magnitude diagram is firmly established due to the good internal precision of the data.

\section{New variables}

A total of 55 candidate variable stars were initially selected from "variability" images processed with the ISIS package. After closer examination, 6 of these turned out to be spurious or uncertain variables and another 13 objects were identified with known RR Lyr stars. The remaining 36 stars are new variables. A preliminary classification was assigned based on the observed light curves, magnitudes and colors. Table 1 lists the equatorial coordinates and the proposed variability class for the new identified variables ${ }^{3}$. The transformation from rectangular to

\footnotetext{
${ }^{2}$ The CCD-based $V / B-V$ color-magnitude diagram published by Côté et al. (1996) was also calibrated with the photographic data from Alcaino \& Liller (1983).

${ }^{3}$ Finding charts as well as complete photometry of all variables are available at the CDS via anonymous ftp to
}

equatorial coordinates was derived based on 297 stars with J2000.0 equatorial coordinates kindly provided by Dr. K. Cudworth (see Peterson \& Cudworth 1994). The rms of the astrometric solution was 0.13 arcsec and we adopt this value for the internal accuracy of the equatorial coordinates derived for variables.

The new variables can be divided into 3 groups: RR Lyr stars, SX Phe stars and other likely shortperiod pulsators, and eclipsing binaries. The proposed classification of the eclipsing binaries follows the convention adopted in the General Catalogue of Variable Stars (Kholopov et al. 1998). In particular, EW stands for contact binaries and EB for eclipsing binaries with $\beta$ Lyr type light curves.

Figure 1 shows $V$-band light curves for all SX Phe variables while light curves of some other variables discussed below are presented in Fig. 2. Preliminary periods could be determined with confidence only for the SX Phe stars. These periods, derived with a programme based on the algorithm described by Schwarzenberg-Czerny (1997), are listed in Table 2 along with average magnitudes $<V>$, peak-to-peak amplitudes $A_{\mathrm{V}}$ and average colors $<B-V>$.

Table 3 gives maximum and minimum magnitudes as well as average colors measured for variables other than the SX Phe stars. Note that for several objects only limits on $V_{\max }$ or $V_{\min }$ are available.

In Fig. 3 we show the positions of the variables listed in Tables 2 and 3 in the $B V$ color-magnitude diagram for the observed field.

cdsarc.u-strasbg.fr $(130.79 .128 .5)$ or via http://cdsweb.u-strasbg.fr/cgi-bin/qcat?J/A+A/373/899. Finding charts are also included in the electronic version of this paper which is available at http://www.edpsciences.org Each chart is 33 arcsec on a side with north up and east to the left. 

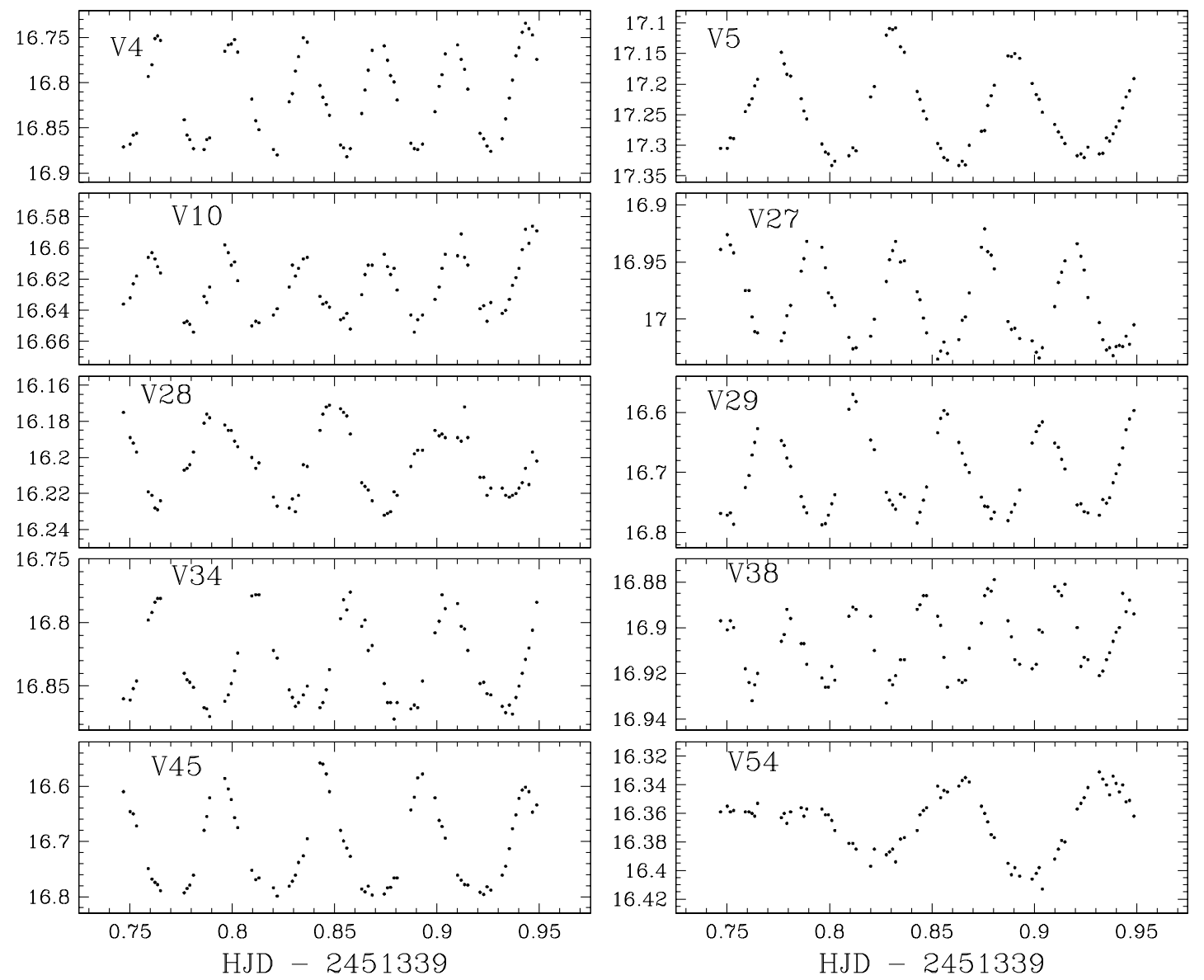

Fig. 1. $V$-band light curves for the SX Phe stars.

\subsection{Pulsating variables}

SX Phe stars are Pop II pulsating variables located in the instability strip below the horizontal branch stars. A recently published catalogue of SX Phe stars in globular clusters (Rodriguez \& Lòpez-González 2000) lists 122 objects in 18 clusters. SX Phe variables are also found in the Galactic field as well as in four dwarf spheroidal galaxies from the Local Group. As observed in other clusters, the SX Phe stars in M 22 are a sub-sample of the blue straggler population. We have examined in detail the light curves of all candidate blue stragglers and have failed to detect any variables besides the objects listed in Table 2. Considering the internal accuracy of the photometry of the detected SX Phe stars, the non-variable blue stragglers marked in Fig. 3 are constant on a time scale of one hour with amplitudes not exceeding 0.01 mag. Mateo (2000; see Fig. 1 therein) notes that in contrast to the RR Lyr stars, most objects located in the dwarf cepheid instability strip are not variable. More dedicated observations would be needed to confirm this in the case of the M 22 blue stragglers or to significantly lower the upper limit on the amplitude of variability. Based on this present data we conclude that the region defined by the limits $16.2<V<17.3$ and $0.45<B-V<0.62$ includes 10 SX Phe variables as well as 19 candidate blue stragglers showing no evidence for variability.

The light curves of variables V5, V28 and V54 seem to be unstable. Multi-modal pulsations are a likely cause of this behavior (e.g. Pych et al. 2001). However, the time base of our observations is too short for any meaningful analysis of power spectra aimed at the detection of possible multiperiodicities in the light curves of these 3 objects.

Our sample of new variables includes 7 RR Lyr stars. Variable V12 is a background object. During our observations it changed its luminosity from $V=16.2$ to $V=16.8$ (see Fig. 2). The remaining 6 variables are likely members of M 22 as their observed luminosities and colors place them on the horizontal branch of the cluster.

Variable V42 was tentatively classified as a pulsating variable based on the asymmetric shape of its light curve. It may be a field $\delta$ Sct star.

\subsection{Eclipsing binaries}

Our sample of eclipsing binaries includes 13 probable contact systems and 3 likely non-contact systems. For most EW candidates the observed light curves cover roughly half of the orbital periods (see for example star V15 in Fig. 2). As can be inferred from Fig. 3, at least some of the contact binaries are field interlopers. In particular, objects located far to the red of the cluster main sequence are unlikely to be members of M 22. Binaries located among the 

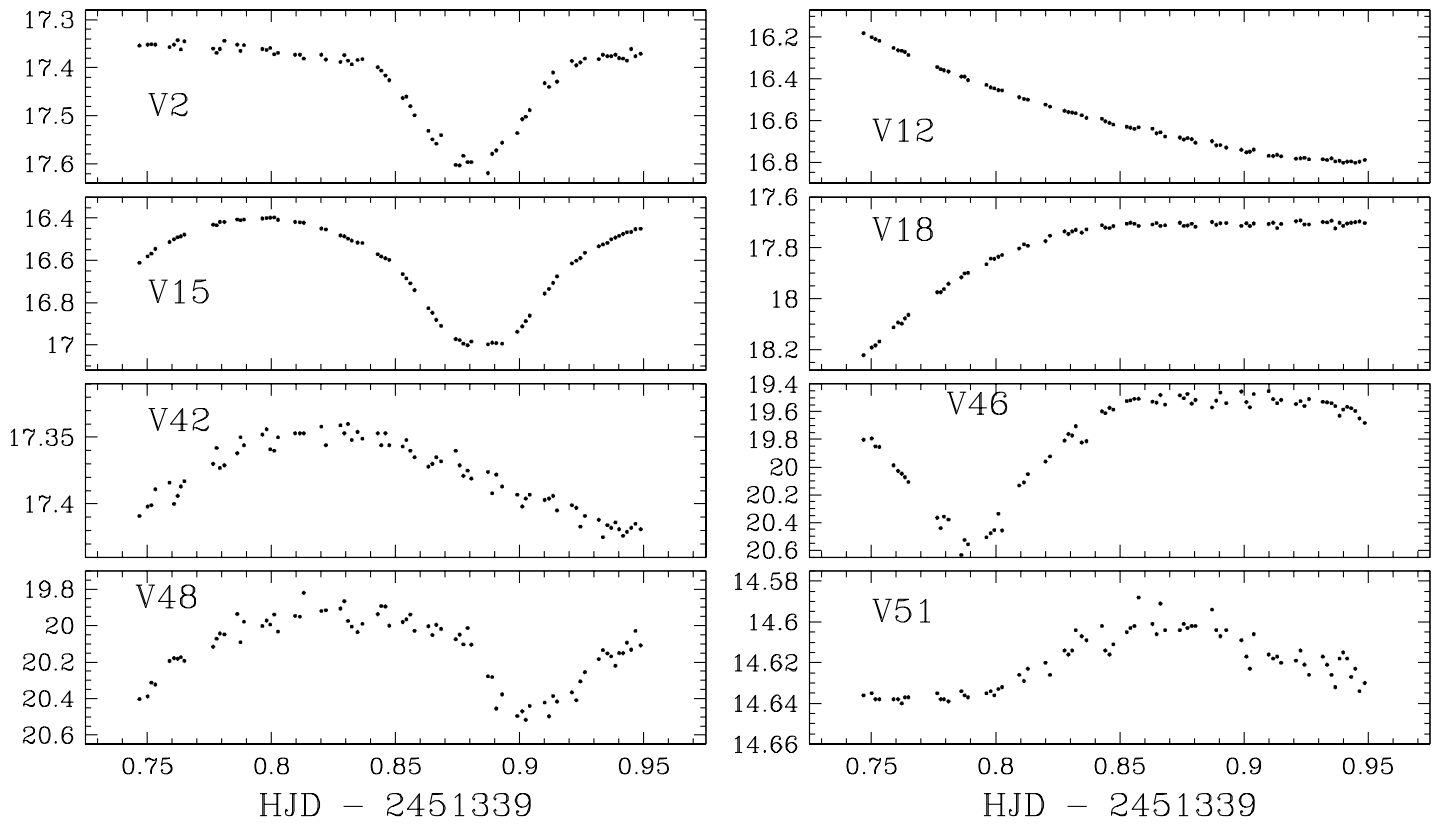

Fig. 2. $V$-band light curves for selected variables from Table 3.

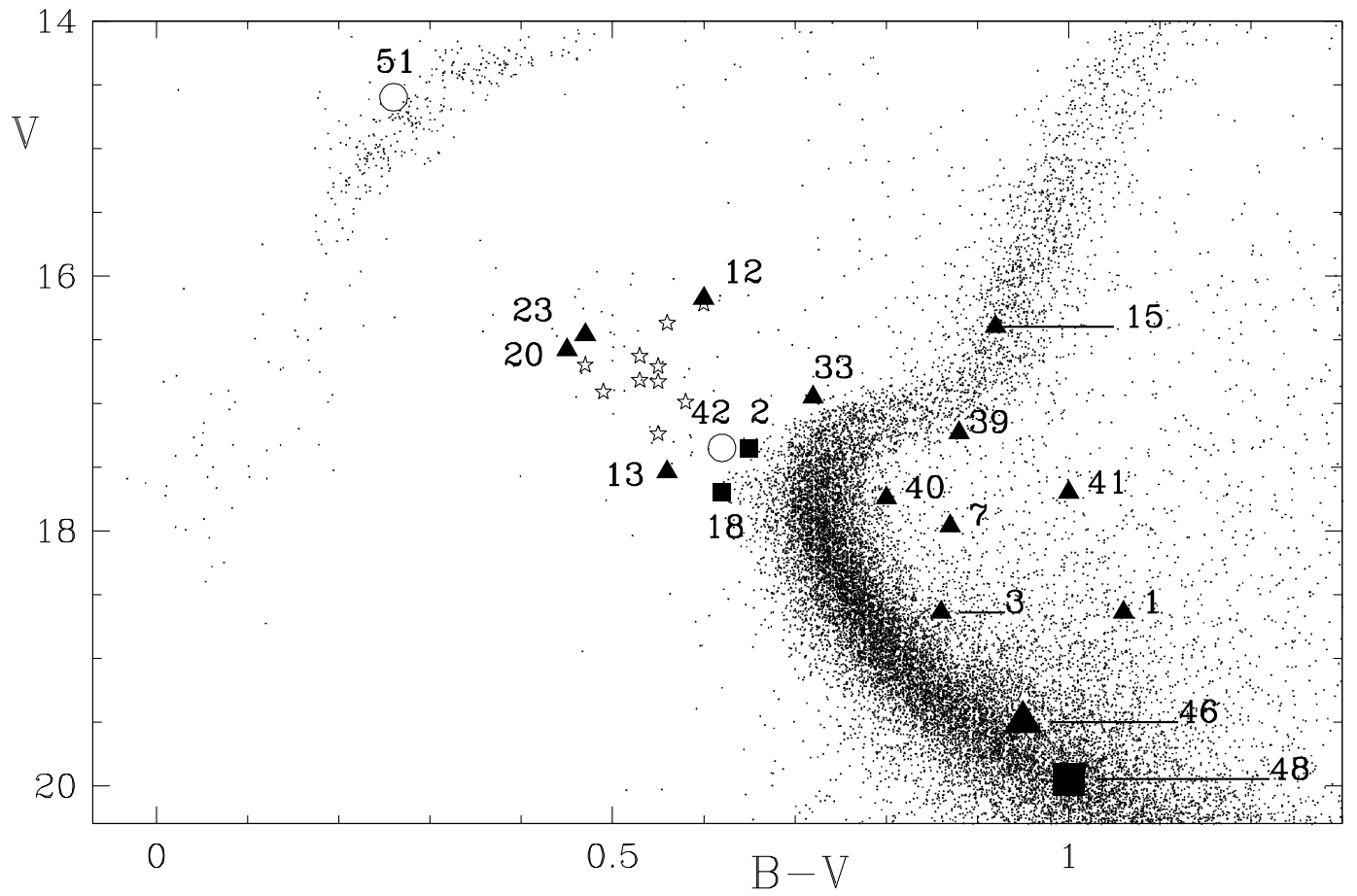

Fig. 3. A color-magitude diagram for M 22 with the positions of some variables. Asterisks denote SX Phe stars, filled triangles denote EW systems, filled squares denote non-contact binaries, and open circles denote variables V51 and V42. SX Phe stars are not labeled.

blue stragglers or close to the cluster main-sequence are possible cluster members. Determination of the periods of these systems would help to assess their membership status (e.g. Rucinski 2000).

Two probable detached systems, stars V2 and V18, are located at the base of the blue straggler sequence in Fig. 3. Their membership status is unclear from these data. The third candidate for a detached binary, star V46, is a relatively faint object located slightly to the red of the lower main-sequence of the cluster.

\subsection{A variable $E H B$ star}

Possibly the most interesting object in the sample is variable V51. This star is located at the top of the extended horizontal branch (EHB) of the cluster (see Fig. 3). Its observed color at maximum brightness $(B-V)=0.26$ 
Table 2. Photometric data for SX Phe stars.

\begin{tabular}{|c|c|c|c|c|}
\hline Name & $P[d]$ & $<V>$ & $<B-V>$ & $A_{V}$ \\
\hline CASE M22_04 & 0.036 & 16.82 & 0.53 & 0.14 \\
\hline CASE M22_05 & 0.061 & 17.24 & 0.55 & 0.22 \\
\hline$C A S E$ M22_10 & 0.037 & 16.63 & 0.53 & 0.06 \\
\hline CASE M22_27 & 0.042 & 16.99 & 0.58 & 0.11 \\
\hline CASE M22_28 & 0.055 & 16.22 & 0.60 & 0.06 \\
\hline$C A S E$ M22_29 & 0.045 & 16.70 & 0.47 & 0.16 \\
\hline$C A S E$ M22_34 & 0.047 & 16.83 & 0.55 & 0.09 \\
\hline CASE M22_38 & 0.033 & 16.91 & 0.49 & 0.05 \\
\hline CASE M22_45 & 0.050 & 16.71 & 0.55 & 0.24 \\
\hline$C A S E$ M22_54 & 0.080 & 16.37 & 0.56 & 0.07 \\
\hline
\end{tabular}

and thus $(B-V)_{0} \approx-0.08$, places it clearly outside the RR Lyr instability strip. The light curve presented in Fig. 2 suggests variability with a period of about $0.2 \mathrm{~d}$ and an amplitude reaching about $0.06 \mathrm{mag}$ in the $V$ band. Very few, if any, variable objects are known among the bright EHB stars $^{4}$. The variability of star V51 may suggest that it is a binary. Saffer et al. (2001) have reported that about $65 \%$ of the sdB stars are spectroscopic binaries. The apparent magnitude of V51, $V \approx 14.6$, makes it a relatively easy target for spectroscopic follow up observations.

\section{Conclusions}

We have reported the identification of 36 new variable stars located in the central field of M 22. The detection of the variables and the measurement of the light curves was accomplished with the image subtraction software developed by Alard \& Lupton (1998). The sample of new variables includes 7 RR Lyr stars, 6 of which are probable cluster members. This result and recent results obtained by our group for some other clusters (M 5, M 55, NGC 6362 and NGC 6934; e.g. Kaluzny et al. (2001) and references therein) indicate that present samples of cluster RR Lyr stars are significantly incomplete even for nearby globulars. Ten SX Phe stars were identified among the cluster blue straggler stars. Approximate periods, average magnitudes and amplitudes are given for these variables. A more detailed study is required to firmly establish their properties. In particular at least 3 variables are candidates for multi-periodic pulsators. More extended data, including radial velocity studies, are also needed to determine which of the 17 detected eclipsing binaries are members of M 22 . Potentially the most interesting result of this quite limited survey is the detection of photometric variability of a star located at the top of the extended horizontal branch

\footnotetext{
4 A small fraction of the subdwarf B stars (sdB stars) exhibit pulsations with periods of the order of a few minutes.
}

Table 3. Photometric data for M 22 variables.

\begin{tabular}{|c|c|c|c|}
\hline Name & $V_{\max }$ & $V_{\min }$ & $(B-V)_{\max }$ \\
\hline CASE M22_01 & 18.64 & 18.78 & 1.06 \\
\hline CASE M22_02 & 17.35 & 17.60 & 0.65 \\
\hline CASE M22_03 & 18.64 & 18.86 & 0.86 \\
\hline CASE M22_07 & 17.96 & 18.01 & 0.87 \\
\hline CASE M22_08 & $<19.9$ & 20.50 & \\
\hline CASE M22_12 & $<16.18$ & $>16.80$ & 0.58 \\
\hline CASE M22_13 & 17.54 & 17.92 & 0.56 \\
\hline CASE M22_14 & 13.93 & $>14.15$ & 0.52 \\
\hline CASE M22_15 & 16.40 & 17.00 & 0.92 \\
\hline CASE M22_16 & $<14.11$ & 14.17 & 0.47 \\
\hline CASE M22_18 & 17.70 & $>18.20$ & 0.62 \\
\hline CASE M22_20 & 16.58 & 16.74 & 0.45 \\
\hline CASE M22_23 & 16.46 & 16.66 & 0.47 \\
\hline CASE M22_26 & $<14.06$ & 14.18 & 0.53 \\
\hline CASE M22_33 & 16.95 & 17.01 & 0.72 \\
\hline CASE M22_36 & 13.99 & 14.33 & 0.46 \\
\hline CASE M22_37 & 14.10 & $>14.22$ & 0.48 \\
\hline CASE M22_39 & 17.23 & 17.40 & 0.88 \\
\hline CASE M22_40 & 17.74 & 17.74 & 0.80 \\
\hline CASE M22_41 & 17.70 & 18.22 & 1.00 \\
\hline CASE M22_42 & 17.35 & 17.42 & 0.62 \\
\hline CASE M22_43 & 18.42 & 18.84 & \\
\hline CASE M22_46 & 19.50 & 20.50 & 0.95 \\
\hline CASE M22_48 & 19.95 & 20.45 & 1.00 \\
\hline CASE M22_51 & 14.60 & 14.64 & 0.26 \\
\hline CASE M22_55 & $<13.87$ & $>14.02$ & 0.55 \\
\hline
\end{tabular}

of the cluster. This object deserves a dedicated follow up study.

Acknowledgements. JK was supported by the KBN grant 2P03D003.17 and by NSF grant AST-9819787. IBT was supported by NSF grant AST-9819787. We thank Dr. Kyle Cudworth for providing astrometric data for the cluster field. Dr. N. Samus kindly furnished equatorial coordinates for the known M 22 variables. We thank Grzegorz Pojmański, Alex Schwarzenberg-Czerny and Basia Mochejska for some useful software. We would like to sincerely thank the anonymous referee for his detailed and very helpful comments on the manuscript.

\section{References}

Alard, C. 2000, A\&AS, 144, 363

Alard, C., \& Lupton, R. H. 1998, ApJ, 503, 325

Alcaino, G., \& Liller, W. 1983 AJ, 88, 1330

Côté, P., Pryor, C., McClure, R. D., Fletcher, J. M., \& Hesser, J. E. 1996, AJ, 112, 574

Clement, C. 1997, AAS Newsl., 84, 15

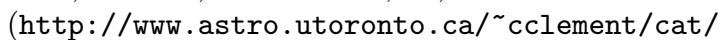
clusters.html) 
Cudworth, K. M. 1990, AJ, 99, 1863

Kaluzny, J., Olech, A., \& Stanek, K. Z. 2001, AJ, 121, 1533

Kholopov, P. N., et al. 1998, Combined General Catalogue of Variable Stars, SIMBAD

Kravtsov, V. V., Samus, N. N., Alcaino, G., \& Liller, W. 1994, AstL, 20, 456

Landolt, A. 1992, AJ, 104, 340

Mateo, M. 2000, ed. L. Szabados, \& D. W. Kurtz, The Impact of Large-Scale Surveys on Pulsating Star Research, ASP Conf. Ser., 203, 187

Paczyński, B. 1997, in The Extragalactic Distance Scale, STScI Symposium, ed. M. Livio (Cambridge: Cambridge University Press), 273
Peterson, R. C, \& Cudworth, K. M. 1994, ApJ, 420, 612

Pych, W., Kaluzny, J., Krzeminski, W., Schwarzenberg-Czerny, A., \& Thompson, I. 2001, A\&A, 367, 148

Richter, P., Hilker, M., \& Richtler, T. 1999, A\&A, 350, 476

Rodriguez, E., \& Lòpez-González, M. J. 2000, A\&A, 359, 597

Rucinski, S. M. 2000, AJ, 120, 319

Saffer, R. A., Green, E. M., \& Bowers, T. P. 2001, ASP Conf. Ser., 226, ed. H. L. Shipman, et al., in press [astro-ph/0012224]

Stetson, P. B. 1987, PASP, 99, 191

Schwarzenberg-Czerny, A. 1997, ApJ, 489, 941

Thompson, I. B., Kaluzny J., Pych, W., et al. 2001, AJ, in press [astro-ph/0012493] 